\title{
Lazarro Spallanzani (1729-1799) et les premières procréations assistées
}

\author{
G. ANDROUTSOS, ST. GEROULANOS \\ Histoire de la Médecine, Faculté de Médecine, Université d'Ioannina, Grèce
}

\begin{abstract}
RÉSUMÉ
Dans cet article sont abordés très brièvement les grands courants concernant la physiologie de la reproduction (ovisme, spermatisme, épigenèse, préformation, génération spontanée, pangenèse) à travers les siècles. En plus, sont présentées les premières expérimentations de procréation artificielle, tout en mettant l'accent sur le rôle de Spallanzani dans ce domaine.
\end{abstract}

Mots clés : Spallanzani, reproduction, procréation assistée, insémination artificielle

\section{INTRODUCTION}

La reproduction des êtres vivants avait longtemps donné lieu à des explications obscures ou fantaisistes qui faisaient volontiers appel à des interventions mystérieuses, voire surnaturelles. Pendant plus de vingt siècles, les observations les plus farfelues, les descriptions les plus ahurissantes font état d'embryons séjourant douze mois dans le ventre, de grossesses masculines, de femmes accouchant de montres ou d'hybrides, d'embryons fabriqués in vitro avec du sperme masculin et du sang menstruel, d'accouchements simultanées de sept ou huit enfants, de superfétation, etc...

\section{LA PHYSIOLOGIE DE LA REPRO- DUCTION A TRAVERS LES AGES}

Pour les présocratiques comme pour Hippocrate (460-377 av. J.-C.), l'embryon résulterait d'un mélange de semences masculine et féminine, qui serait " cuit " par la matrice féminine ou « coagulerait » pour former l'embryon. Mais avec Aristote (384-322 av. J.-C.), tout change : l'homme et la femme ne se partagent plus la responsabilité de l'acte procréateur. Il voit dans l'embryon le résultat d'une résistance de la matière (qui provient de la femme) à la forme (issue de l'homme). L'idée de l'embryon se résume tout entière dans le sperme de l'homme, qui est presque animé, la femme n'étant que nourricière [2].

Selon une autre théorie, l'enfant est entièrement produit par sa mère. Celle-ci possède dans son ventre un organe semblable à un petit vase qui, à chaque rapport sexuel, se remplit peu à peu de sperme. Lorsque le vase est plein, il déclenche la fabrication d'un bébé. Mais le fournisseur du sperme n'a pas plus de rôle dans la constitution de ce bébé. Il a provoqué un événement, mais n'y a pas participé. Une telle vision est en accord avec celle développée durant le XVIIe siècle par les "ovistes " qui, à la suite de William Harvey (1578-1657), soutenaient que le foetus est préformé dans l'ovule et prétendaient que tout vient de l'œuf qui se développe peu à peu. Harvey, en 1651, lors de la publication à Londres de son ouvrage Exercitationes de generatione animalium, où il propose cette théorie, ne disposait pas de microscope. Quelques années plus tard, Marcello Malpighi (1628-1694) systématisa l'emploi de cet instrument qui venait d'être inventé par Antony van Leeuwenhoek (1632-1732) et aboutit à la même conclusion que Harvey [15].

Correspondance : G.Androutsos, 1, rue Ipirou, 10433, Athènes, Grèce 
Jusqu'au XVIIIe siècle, les conceptions aristotéliciennes et hippocratiques, relayées par Claude Galien (131-201 apr. J.-C.) puis par les médecins arabes, règnent sur l'embryologie. La plupart des penseurs voient dans la semence un surplus de nourriture. Ce qui permet d'expliquer que les enfants ne puissent procréer, puisqu'ils ont besoin de tous les aliments qu'ils ingèrent pour croître et n'ont donc pas de "surplus ». En général, tous s'accordent pour clamer l'évidente infériorité de la femme. Si le couple est stérile, c'est assurément que la matrice de la femme est inadéquate [11]. En 1677, Leeuwenhoek, utilisant des lentilles grossissantes, découvrit dans le sperme des insectes, des chiens et des hommes, les spermatozoïdes. Pour notre espèce, il les nomma " homuncules": ces petits êtres sont vivants, ils contiennent le futur homme ou la future femme. Dans ses articles il représente des bébés occupant la tête du spermatozoïde. Leeuwenhoek, découvrant les spermatozoïdes se serait laissé emporter par son enthousiasme. Il croit observer de véritables familles d'homuncules. Il distingue sur les lames de son microscope des spermatozoïdes mâles et des spermatozoïdes femelles, et, à la saison des amours, il voit de petits spermatozoïdes accompagnant leur mère. Longtemps, les «animalculistes» ont triomphé. La science donnait ainsi raison à la thèse de Platon (427-347 av. J.-C.) qui, dans le processus de procréation, attribuait le rôle essentiel au mâle [11]. La querelle entre « ovistes » et «spermatistes " fut alors animée, car l'enjeu était d'importance : descendons-nous de notre père ou de notre mère ? Cependant, si elles étaient en désaccord sur la localisation initiale de l'individu, les deux théories convergeaient sur l'essentiel : cet être est entièrement préformé, ses divers organes sont déjà présents ; il se contente de les développer au cours de la gestation. Ces deux théories aboutissaient l'une et l'autre à la même conclusion nécessaire : l'emboîtement des générations successives. Le garçon présent dans le spermatozoïde pour les spermatistes (la fille présente dans l'ovule pour les ovistes) possède déjà des testicules (des ovaires), dans lesquels existent les spermatozoïdes (les ovules) qu'il (elle) émettra au cours de sa vie. Certains d'entre eux contiennent les enfants auxquels il (elle) donnera naissance. Et les enfants euxmêmes, déjà préformés, possèdent en eux leurs propres enfants. Chaque homme et chaque femme contiennent ainsi en eux la totalité de leurs futurs descendants jusqu'à la fin des temps [15].

Au cours du XVIIIe siècle, il est peu à peu devenu évident que spermatisme comme ovisme étaient des théories insoutenables. $\mathrm{La}$ notion de préformation, présentant l'embryon comme une réduction du futur adulte, dut être abandonnée lorsque Fiedrich-Caspar Wolff (1733-1794) [36] démontra qu'au cours du développement les tissus, d'abord indifférenciés, peu à peu se spécialisent et réalisent les divers organes. Ceux-ci n'etaient pas déjà présents dans l'embryon, prêts à se développer pour atteindre un jour leur dimension définitive ; ils résultent au contraire de la transformation de structures initiales toutes identiques. D'autre part, il est clair que l'être procréé a des ressemblances avec ses deux géniteurs et non avec un seul d'entre eux. Mais une fois récusées les thèses spermatiste et oviste, que dire? Le mystère paraît si profond qu'il a longtemps défié l'imagination des scientifiques. Dès le milieu du XVIe siècle, Ambroise Paré (15091590) [21] pose le problème avec une merveilleuse clarté. Il admet une certaine symétrie des rôles des deux géniteurs. Il soulève, aussi, le problème de la ressemblance entre parents et enfants sans proposer de solution. La fonction de l'ovaire - ce "testicule " des femelles vivipares qu'étudia Nicolas Stenon (1638-1686) - fut entrevue par Reinier de Graaf [10]: il acheva en 1673 la description du follicule à évolution cyclique auquel son nom demeure attaché. Comme Johann van Horne (1668) [3] et Theodor Kerckring (1671) [16], il confondit toutefois l'enveloppe de l'ovule avec l'œuf lui-même. Simultanement (1677), Jean Ham [20] et Antoine Vallisneri [34], puis Niklaas Hartsoecker (1656-1725) [13], démontraient l'existence et le rôle des " animalcules » (spermatozoïdes). Ils notaient également le mécanisme de la nidation de l'œuf. Au terme de ses observations sur les daines, William Harvey (1578-1657) [14] énonçait déjà en 1651 le principe fondamental : "Ovum esse primordium commune omnibus animalibus ". Cette notion nouvelle devait être aussitôt développée 
par Francesco Redi, d'Arezzo [25], qui dans son ouvrage de 1684 niait déjà tout phénomène de génération spontanée. Anton van Leeuwenhoek (1632-1723) [6] parvint aussi à démontrer par des expériences rigoureuses " qu'aucune créature ne peut prendre naissance sans génération ». En 1757, Albrecht von Haller (1708-1777) [35] pouvait dire : " Personne ne croit plus à la génération spontanée ». Au XVIIIe siècle, les explications du processus de fécondation sont aussi dogmatiques que fantasques. Épigénistes et préformationnistes se combattent à coup d'arguments idéologiques. Les premiers considèrent que l'embryon prend forme et se structure étape par étape, en fonction de l'environnement. Les seconds voient en lui un homme miniature, un homuncule, déjà tout entier formé. François de Plantade [31] à Montpellier va même jusqu'à publier l'observation suivante : «Examinant un des plus grands spermatozoïdes, je le vis se dépouiller de son enveloppe et il montra nettement à nu ses deux jambes, ses cuisses, sa poitrine, ses deux bras. Sa dépouille tirée vers le haut le coiffait à la façon d'un capuchon. Spectacle certes admirable et incroyable " et il termine par " les caractères distinctifs des deux sexes n'ont pu être reconnus à cause de leur exiguité ». Mais Georges-Louis-Leclerc Buffon (1707-1788) [5] réfute catégoriquement l'existence de l'homonculus : "Comment ce petit personnage pourrait-il être plus formé que ne l'est le fotus à un mois ! ». Ces observations fantaisistes jettent un certain discrédit sur l'animalcule. L'ovisme regagne du terrain. La découverte à la fin du XIXe siècle, des gamètes mâles et femelles, a compliqué le débat au lieu de l'éclairer. Les uns, à la suite de François de Plantade, affirment que l'embryon provient à part entière du spermatozoïde ; les autres, à l'instar de Charles Bonnet (17201793) [4], défendent la thèse oviste : tout est contenu dans l'ovule, et l'animalcule spermatique n'est porteur que de l'aura seminalis, principe qui permet au germe contenu dans l'ovule de se développer. Aux thèses privilégiant l'homme ou la femme s'ajoutent celles des moléculistes, qui reprennent l'hypothèse hippocratique du mélange des semences en l'adaptant à la physique moderne : chaque partie du corps de l'homme et de la femme partici- pe à l'élaboration de la semence [3]. Le principal défenseur de cette théorie est sans doute Buffon qui imagine la théorie de la "pangénèse $»$ : chaque partie du corps des deux géniteurs envoie dans les "liqueurs séminales" un messager porteur de la recette de fabrication de cette partie. Au moment de la rencontre, les liqueurs se mélangent ; les messagers correspondants se cherchent, se trouvent, fusionnent et réalisent un messager qui construit l'organe correspondant de l'enfant. Cette fusion permet de comprendre pourquoi chaque caractéristique de l'enfant est proche de la moyenne de celles de ses parents : « Je crois donc que la liqueur séminale du mâle répandue dans le vagin et celle de la femme répandue dans la matrice sont deux matières également actives, également chargées de molécules organiques propres à la génération... » [5]. Cette théorie des " molécules organiques » fut assez largement acceptée. En 1740, Charles Bonnet [4] avait découvert que les oufs des pucerons pouvaient se développer sans la participation des mâles (parthénogénèse). Ainsi donc, la capacité des œufs à se développer pouvait être déclenchée par quelque force générative exercée par la femelle elle-même. Ce fut une découverte surprenante pour les contemporains de Bonnet, mais les recherches du XIXe siècle montrèrent que ce type de "naissance virginale était très répandue dans le règne animal. Dans les années 1840, un type particulier de parthénogénèse, " l'arrhénotoquie ", fut découvert chez les hyménoptères, dans lequel un œuf non fécondé donne naissance à un mâle haploïde. Cet extraordinaire phénomène fut décrit pour la première fois par Johann Dziezon [19].

Pas plus que le médecin antique, le savant des Lumières ne connaît l'origine de la semence, ni la part que prennent respectivement la femme et l'homme dans la reproduction. En 1841, Georges Cuvier (1769-1832) [8] affirmera encore que les « vers spermatiques » ne sont que des microzoaires. Des autres scientifiques y voient des parasites qui empêchent par leur mouvement le sperme de " coaguler " et de produire un embryon [11]. Le rôle du spermatozoïde ne fut définitivement démontré qu'en 1824 par les expériences de Jean-Louis Prevost (1790-1850) et Jean-Baptiste-André Dumas (1800-1884) 
qui publièrent les résultats d'une série d'expériences ingénieuses et décisives sur les grenouilles : celles-ci montraient, de manière concluante, que les spermatozoïdes représentaient l'élément fécondant, et que le liquide séminal n'était qu'un vecteur [23]. En 1841, Calister démontre que l'animalcule dérive des cellules du testicule et quelques années plus tard le spermatozoïde est reconnu comme cellule à part entière avec son noyau [17]. En 1843, Barry Martin (1802-1855) [18] avec le lapin, et, en 1851, George Newport [19] avec la grenouille, démontrèrent la présence de spermatozoïdes à l'intérieur des œufs fécondés, bien qu'aucun d'entre eux n'ait pu observer la pénétration effective d'un spermatozoïde dans un œuf, ni en décrire le devenir. Leurs observations ne permirent donc pas de mettre en échec les théories physicalistes de la fécondation. En 1854, Thuret [19] montra que, chez Fucus, des spermatozoïdes ciliés entouraient l'œuf et y pénétraient. Il fut même capable de réaliser des fécondations artificielles. La première description concluante du processus de fécondation fut fournie en 1856 par $\mathrm{N}$. Pringsheim [19] chez l'algue d'eau douce Oedogonium. Il observa réellement l'entrée du gamète mâle dans l'oogone femelle, et tira de cette observation la conclusion correcte que la première cellule (le zygote) du nouvel organisme était formée par la fusion du gamète mâle avec la cellule-œuf femelle, et que la fécondation était réalisée par un seul spermatozoïde. Cette observation resta elle aussi ignorée, en dépit de son importance capitale. Â la fin du XIXe siècle les tenants de la «conception électrique" (le coït vu comme une excitation électrique qui provoque la croissance de l'œuf, niché dans la matrice féminine) sont encore nombreux. Trente ans plus tard, les mécanismes de la fécondation seront élucidés.

La religion, qui interdit des recherches sacrilèges, incita les savants à faire l'ombre sur certaines de leurs réussites. Au lendemain des expériences de Spallanzani, John Hunter pratique une insémination sur l'épouse d'un homme hypospadique [1]. Malgré le succès de l'opération, Hunter se garde bien de la mentionner dans ses écrits, et seul le bouche à oreille en avertit ses confrères. Un siècle plus tard, rien n'a changé. Le Dr Gigon [11], méde- cin des hôpitaux d'Angoulême, attend vingt et un ans avant de faire état d'une insémination qu'il aurait réussie en 1846. En un temps où les mécanismes de la fécondation ne sont pas élucidés, une telle expérience montre que le coït n'est pas indispensable à la conception. En 1875, Hertwig [3] observe et décrit la pénétration du spermatozoïde dans l'ovule de l'oursin, ouvrant ainsi la voie à la procréation artificielle. En 1890 enfin, un médecin, le Dr Dickinson [11], ose l'impensable : pratiquer une insémination avec sperme de donneur. Pour la première fois, les enfants adultérins entrent dans le champ de la médecine, et de la vie conjugale.

\section{LA VIE DE SPALLANZANI}

Lazzaro Spallanzani, physiologiste, naturaliste, physicien, prêtre et écrivain Italien, naquit à Scandiano, dans la région de Modène, le 12 janvier 1729 ; il fit ses humanités chez les jésuites de Reggio nell'Emilia, reçut les ordres mineurs, enseigna à Reggio même, puis à Modène. Il fut poussé vers l'étude des sciences naturelles par sa cousine, la célèbre Laura Bassi, que son sexe n'empêcha pas d'occuper la chaire de physique de l'Université de Bologne. Spallanzani fut appelé par Marie-Thérèse, impératrice d'Autriche, à la chaire d'histoire naturelle de l'Université de Pavie en 1759 où il fit presque toutes ses expériences. En 1781, il entreprit un grand voyage sur les côtes orientales de la Méditerranée. Il fut à la base de travaux importants en physique, en chimie, en météorologie, en vulcanologie et en physiologie. Parmi ses nombreuses activités scientifiques, il s'attacha à l'étude de la digestion, d'abord sur les oiseaux, et ensuite sur des mammiferes et sur lui-même, et démontra ainsi l'action chimique du suc gastrique [7]. Il s'intéressa également à la physiologie de la respiration, de la circulation du sang, et au pouls. Mais il fut avant tout l'adversaire de la théorie de la génération spontanée et Pasteur avait, dans son appartement, un tableau (Fig. 1) représentant le naturaliste italien, la main posée sur son bureau où se trouvait le microscope (Fig. 2) qui lui permit de se faire le défenseur de la non-spontanéité des germes. Par de nombreuses expériences, Spallanzani démontra que la fécondation nécessite un contact matériel entre le spermatozoïde et 


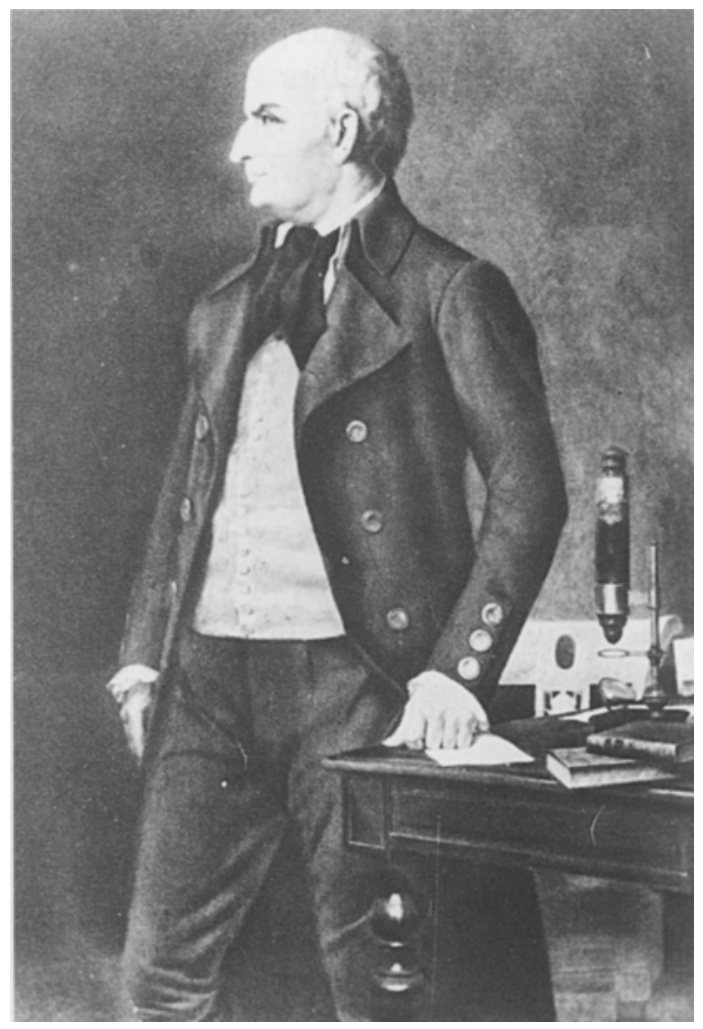

Figure 1 : Portait de Lazarro Spallanzani

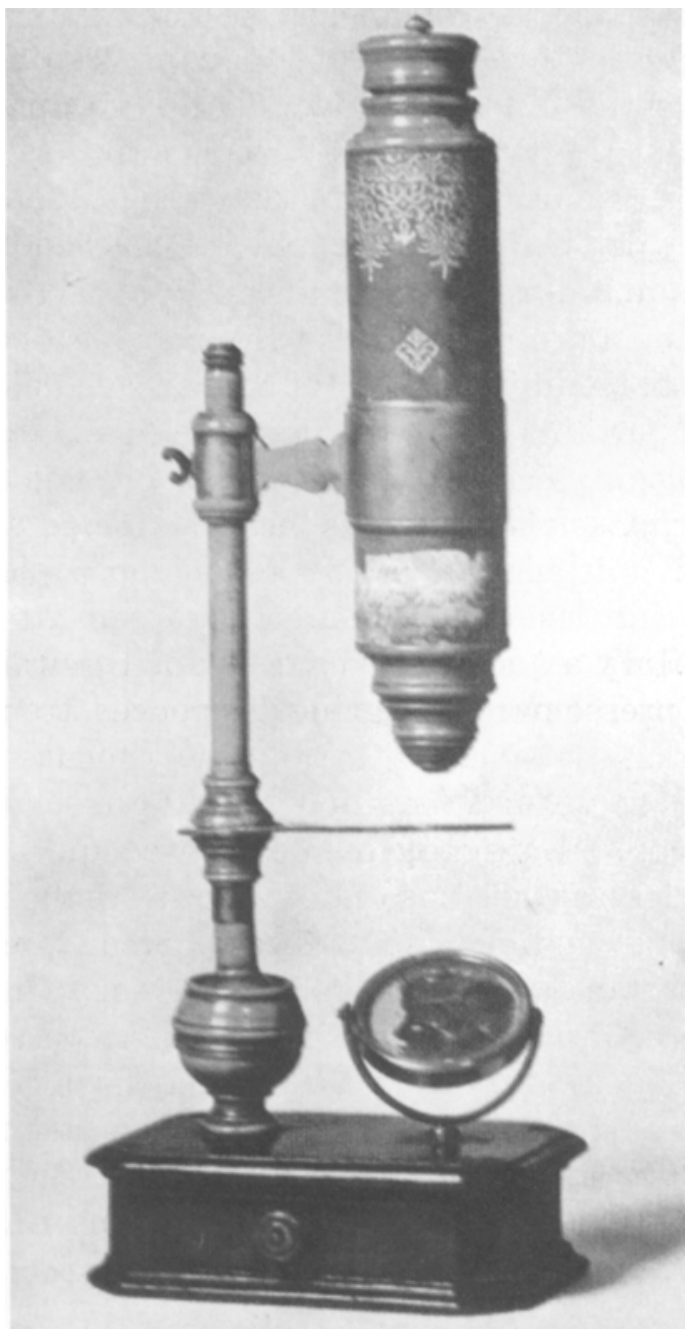

Figure 2 : Le microscope de Spallanzani 
l'ovule. En 1777, il obtint la première fécondation artificielle en arrosant des œufs de semence de crapaud mâle, puis il réussit une fécondation artificielle chez une chienne [26]. Après la prise de Pavie, le 26 mai 1796, par les troupes françaises, Salicetti, homme politique français détaché auprès de l'armée d'Italie, lui offrit, de la part du Directoire, une chaire à l'Université de Paris, qu'il refusa. Il mourut à Pavie, en février 1799, d'un cancer prostatique.

\section{LES PREMIÈRES EXPÉRIMENTATIONS SUR LA PROCRÉATION DES GRENOUILLES}

La grenouille tient une place de choix dans les laboratoires car on peut étudier sur elle les problèmes essentiels de la division, de la différenciation, de la vie, et de la survie cellulaire. De surcroît, elle procure à l'embryologiste un matériel vraiment privilégié : les dimensions de son œuf, ni trop gros ni trop petit, son mode de fécondation externe, les traits de développement larvaire sont autant de conditions idéales pour une expérimentation sur elle. Ce n'est pas un hasard si elle fut à l'origine de tant de découvertes, si tant de fois elle a montré la route féconde, donné lieu à l'expérience décisive, au point qu'on peut dire sans exagération que toute l'histoire de l'embryologie expérimentale passe par elle [28].

En 1672, Jan Swammerdam (1637-1684) [32] fut le premier qui se prononça en faveur de la fécondation externe chez les grenouilles et il figura une gerbe liquide jaillissant du mâle dans son " Miraculum naturae...". Leeuwenhoek, plus tard, avait remarqué que le mâle saisissait la femelle avec ses pattes et restait cramponné à elle pendant des jours sans posséder pourtant d'organe copulateur. Selon lui : « le soi-disant accouplement des grenouilles et leur habitude de saillie sert à permettre au mâle, à mesure que la femelle émet ses ovules, de déposer en même temps sa semence " [6]. En 1724, Frédéric Mentzius, professeur à l'Université de Leipzig, imagine que " pendant l'accouplement la liqueur séminale sort de la proéminence charnue qu'on observe au pouce du mâle, qu'elle entre par mille chemins inconnus dans l'ovaire pour en féconder les œufs » [33]. Buffon [5] soutiendra la même idée à propos des ergots du coq, "Le coq a... deux verges !... elles agissent toutes deux ensemble au cours du coït, qui est au moins une forte compression si ce n'est un vrai accouplement. C'est par ce double organe que le coq répand la liqueur séminale dans la matrice de la poule .

\section{REAUMUR ET LA PROCREATION DES GRENOUILLES}

En 1736, Réaumur (1683- 1757) [24] avait écrit : " J'ai fait bien des tentatives avant que de parvenir à savoir donner à des grenouilles mâles des culottes satisfaisantes. La vessie est l'étoffe que j'ai employée d'abord, mais elle devient trop molle, trop flasque dans l'eau ; elle s'y chiffonne aisément ce qui m'eût laissé incertain si le derrière était assez couvert. Le taffetas ciré m'a paru plus convenable, mais après en avoir fait des culottes, les avoir mises, les grenouilles s'en sont départies devant moi... Je suis parvenu à leur donner des culottes dont elles ne peuvent se défaire... ce qui assure le tout, c'est que j'ai donné des bretelles à ces culottes » et avait décrit l'accouplement de grenouilles de la façon suivante : «...On a donné une culotte de vessie et une culotte bien fermée qui surtout bouche le derrière à une grenouille mâle accouplée de la veille. Son accouplement n'en a pas été dérangé ; elle continue d'être posée sur la femelle... Cette expérience doit m'apprendre des faits bien curieux... Ma première paire de grenouilles accouplées n'a aucunement changé de place depuis hier matin... Le vrai accouplement des grenouilles se ferait-il par les doigts? Mais s'il n'y a aucune ouverture dans l'endroit qui est pressé par le chagrine des pouces, trouverait-on quelque chose dans l'organisation des jambes qui fit croire qu'une matière séminale est portée au chagrine des pouces, qu'elle y est filtrée et qu'elle se filtre au travers de la peau ? " Et continue : « J'ai observé avec attention l'endroit de la femelle contre lequel les mains du mâle pressent et je n'ai rien aperçu qui eût apparence d'ouverture ». Les pouces des mâles présentent, en effet, des excroissances rugueuses qui augmentent l'adhérence. Ces excroissances semblaient correspondre à « des assemblages glandulaires ». Pour vérifier 
ces « conjectures bien singulières ", Réaumur envisagea de faire porter des gants en taffetas ciré à quelques mâles et mit l'accent sur la brièveté de ce moment critique pour l'observateur. Réaumur, en voulant observer une ponte mit douze couples de grenouilles dans douze poudriers de verre. Il en garda huit et donna deux des quatre restants à sa collaboratrice. Un matin, sa collaboratrice constata qu'une de ses grenouilles venait de pondre et décida de porter une extrême vigilance aux grenouilles de la paire restante. Selon le récit de Réaumur : "Son attention à les considérer fut récompensée au bout d'un quart d'heure... elle aperçut les œufs qui commencèrent à sortir... elle tourna les yeux vers le derrière du mâle comme je le lui avais demandé... elle en vit sortir un jet, qu'elle n'a su comparer à rien de plus ressemblant qu'à un jet de fumée de pipe... estce que le mâle ne ferait sortir de son corps qu'une liqueur réduite en vapeur ? » [12].

\section{SPALLANZANI ET LES PREMIÈRES FÉCONDATIONS IN VITRO}

La première fécondation in vitro fut essayée pour la première fois sans succès en 1680 par Swammerdam [32] avec des cufs et spermes de poisson. En 1742, Ludwig Jacobi [31] y réussit en effectuant des fécondations sur les poissons. Il avait pris des truites femelles à l'époque du frai, et, en pressant leurs flancs, avait fait tomber dans l'eau des ovules mûrs. Il avait ensuite arrosé de laitance les ovules en procédant de la même façon avec des truites mâles. Les œufs furent fécondés et des alevins naquirent quelques semaines plus tard.

Le 8 août 1767, Charles Bonnet écrivit à son ami Spallanzani, pour lui faire part d'une idée que l'expérience de Jacobi lui suggérait : " $\mathrm{Ne}$ pourriez-vous pas essayer de féconder les œufs de grenouilles sans l'intervention du mâle ?... Il faudrait tenter d'extraire des vésicules du mâle la liqueur prolifique et d'en arroser un certain nombre d'œufs ». Bonnet avait aussi mentionné dans son livre Considérations sur les corps organisés une expérience que Malpighi avait réalisé sur le ver à soie en 1669 .
Il avait essayé de féconder des œufs en les arrosant avec la liqueur de mâles [4]. Spallanzani, en reprenant les expériences sur les batraciens, allait démontrer le rôle de la semence mâle dans la fécondation des œufs. En effet, dès le 16 mars 1677, Spallanzani après avoir baigné des œufs femelles dans la "liqueur » provenant des vésicules séminales d'un mâle fraîchement sacrifié, obtint la naissance de larves. Spallanzani aussi avait montré que la semence gardait son pouvoir fécondant même si elle était conservée trentequatre heures dans une glacière. Avec ses expériences, Spallanzani, put confirmer tout d'abord les dires de Leeuwenhoek : les grenouilles mâles ne possédaient pas d'appareil copulatoire. Les œufs prélevés dans l'abdomen de femelles accouplées demeuraient stériles : la fécondation ne semblait donc pas interne dans cette espèce. Pour mieux le démontrer, Spallanzani allait affubler les mâles de petits caleçons en taffetas ciré, comme l'avaient déjà fait Réaumur et son collaborateur, l'abbé JeanAntoine Nollet (1700-1770) [12]. Mais Spallanzani, contrairement à ses prédécesseurs, trouva à l'intérieur du caleçon des gouttelettes de liqueur séminale. Il mit dans un verre de montre, un peu moins de onze grains de la liqueur séminale de plusieurs crapauds. Dans un autre verre semblable, mais un peu plus petit, il plaça vingt-six têtards (œufs de grenouille) qui par la viscosité de la glu, s'attachèrent avec ténacité à la partie concave du verre. Cette cupule renversée fut superposée à celle qui contenait la semence pendant cinq heures. Spallanzani répéta cette expérience en diminuant autant que possible la distance entre la semence et les cufs. Les conditions étaient remplies pour que l'aura seminalis, si elle existait, allât du sperme aux œufs placés à une si courte distance au-dessus de lui. Pourtant la fécondation n'eut jamais lieu dans ces circonstances. Au contraire tout contact matériel entre le sperme et les œufs produisait leur fécondation. Si Spallanzani prenait, avec la pointe d'une aiguille, une goutte de sperme, et la mettait en contact direct avec des œufs, la fécondation avait lieu [12]. 


\section{SPALLANZANI ET LES PREMIÈRES INSÉMINATIONS ARTIFICIELLES RÉUSSIES}

Alors qu'il avait fait la preuve du pouvoir des animalcules, Spallanzani, malheureusement, oviste convaincu et préformationiste ne discerne pas le rôle des spermatozoïdes. Après ses succès sur les grenouilles, Spallanzani, en 1781, pratiqua la première insémination chez une chienne qu'il avait au préalable isolée dans une chambre pour assurer son abstinence. Lorsque la chienne manifesta ses chaleurs, Spallanzani injecta dans sa matrice de la semence d'un chien de la même race. Après soixante-deux jours, la chienne mit bas trois chiots. «Le succès de cette expérience me fit un plaisir, écrivit Spallanzani, que je n'ai jamais éprouvé dans aucune de mes recherches ». Bonnet, félicita son ami, par une lettre datée du 13 janvier 1781 : «C'est là une des plus grandes nouveautés qui se soient offertes aux yeux des naturalistes depuis la création du monde... Je ne sais même pas si ce que vous venez de découvrir n'aura pas quelque jour dans l'espèce humaine des applications auxquelles nous ne songeons pas et dont les suites ne seront pas légères... »[4]. Le pressentiment de Bonnet allait se révéler juste. En 1791, le celèbre chirurgien écossais John Hunter (17281793), obtenait après insémination, une grossesse chez la femme d'un drapier qui présentait une malformation du pénis, un « hypospadias " lui interdisant tout coït normal. Cet état pathologique avait amené Hunter à proposer au couple un artifice, l'introduction du sperme au fond du vagin à l'aide d'une seringue ordinaire. Cette «première » chez l'humain conduisit à une naissance [12].

\section{LES ERREURS THÉORIQUES DE SPALLANZANI}

Mais, si le rôle fécondant de la semence apparaissait sans équivoque, on était loin encore en cette fin du XVIIIe siècle, de pénétrer dans l'intimité du phénomène ! Spallanzani connaissait mieux qu'un autre, pour les avoir longuement observés sous le microscope, les animalcules spermatiques, les spermatozoïdes, qui grouillent dans la liqueur séminale de la grenouille; mais le grand physiologiste se refusait à leur attribuer le moindre rôle dans la fécondation de l'oeuf, qui, selon lui - et conformément à la thèse classique de l'ovisme - contient le petit foetus en miniature. A vrai dire, les arguments de Spallanzani ne manquaient point de force persuasive : ne voit-on pas, après la fécondation, l'oeuf se déformer, se modeler, pour se convertir en têtard ? La continuité n'est-elle pas évidente entre le germe femelle et l'embryon ? A cette époque, où la notion de cellule n'était même pas ébauchée, il était bien difficile, sinon impossible, de concevoir quelle manière de collaboration pouvait exister entre un oeuf et un animalcule spermatique, entre une boule relativement volumineuse et un microscopique filament [28]. C'est ainsi que Spallanzani allait paradoxalement rejeter ce qu'il avait pourtant magistralement démontré, à savoir le rôle des animalcules dans la génération. Trompé par les moyens d'observations dont il disposait, il crut qu'au cours de l'évaporation d'une goutte de sperme, les animalcules se rassemblaient au centre de la goutte et disparaissaient de ses bords (Spallanzani avait aussi constaté qu'il suffisait de filtrer le sperme sur des morceaux de tissus, pour lui faire perdre son pouvoir fécondant). A deux reprises, il utilisa cette "semence sans vers " selon lui et aurait obtenu pourtant des oeufs fécondés. En outre, il aurait constaté que la fécondation survenait même si « deux ou trois grains» de semence étaient dilués dans « douze à dix-huit onces » d'eau. «Avec une telle dilution, il n'y a plus d'animalcule ! » pensait-il. Que la fécondation puisse survenir, alors que dans la solution fécondante aucun animalcule n'ait été aperçu, amenait Spallanzani à la conclusion suivante : "La longue habitude que j'ai d'étudier les vers spermatiques, soit de l'homme, soit des animaux, me persuade que je ne me suis pas trompé dans ces expériences délicates, ce qui démontre la fausseté du système de Leeuwenhoek et de ses disciples " [12]. Spallanzani élimina ainsi le rôle fécondant des animaux spermatiques et l'aura seminalis. Selon les partisans de l'aura seminalis l'œuf était fécondé par une vapeur mystérieuse qui se dégageait par le mouvement des animaux spermatiques, la matière de l'embryon étant fournie par l'organisme femelle. Spallanzani affirmait à propos des spermatozoïdes : «Ces 
animalcules ne sont pas des petits hommes !» Selon une lettre écrit à Bonnet : " La liqueur spermatique sera donc pour moi le fluide stimulant qui, en pénétrant le coeur du têtard, le déterminera à battre plus fréquement et plus fort ". Cette liqueur est aspirée par les pores, les "petites bouches" qui criblent la peau de l'oeuf. Elle parvient au coeur du fœtus qu'elle met en mouvement. Si cette hypothèse est juste, peut-être existe-il d'autres substances capables de stimuler le coeur foetal ? [30]. Bonnet, à son tour, avait suggéré à Spallanzani, en 1768, de réaliser des expériences de parthénogénèse : « Je ne veux pas vous cacher une vision que j'ai eue... Je voudrais que vous essayassiez de substituer le fluide éléctrique à la liqueur séminale des grenouilles pour féconder les œufs "Sur les conseils de Bonnet, Spallanzani essaya de soumettre des oeufs vierges, soit au courant éléctrique, soit à l'action de diverses substances telles que le vinaigre, l'esprit de vin, des acides, du sang, de l'urine. Ces premières tentatives de parthénogénèse expérimentale échouèrent. Cette expérience fut finalement réussie en 1899 par Jacques Loeb (1859-1924). En immergeant des œufs vierges d'oursins dans de l'eau de mer additionnée de chlore et de magnésium, il fit naître des larves [27].

\section{SPALLANZANI : « LE SPERMATO. ZOÏDE EST UN PARASITE»}

Si l'animalcule procréateur ne suscite qu'indignation et répugnance, l'animalcule parasite éveille un sentiment de sympathie. Spallanzani ne se lasse pas d'admirer le spectacle du sperme. "Eussions-nous soupçonné, s'exclame-t-il, que cette liqueur précieuse qui est le principe reproducteur des grands animaux, étoit en même temps l'élément destiné à la nourriture et aux plaisirs d'une multitude innombrable de très petits êtres vivants ?" L'abbé Spallanzani idéalise ces insectes. Ils sont des créatures d'une "sagesse suprême ", d'une « sagesse adorable qui s'est plu à multiplier les êtres sentants, et à ne laisser déserte aucune portion de la nature ". Pour Spallanzani, les animalcules sont aveugles car leur déplacement est plus incohérent que ne le dit Albrecht von Haller (1708-1777). La vue, dans ce séjour obscur, leur serait d'ailleurs inutile: "Les animaux se heurtent contre tous les obstacles ; quand ils sont au milieu d'eux, ils s'agitent, ils y font mille contorsions jusqu'à ce qu'ils en soient dehors, ils suivent enfin la route où ils éprouvent la plus petite résistance ». Parfois, la queue de plusieurs animalcules s'entortille et on les voit s'agiter dans l'espoir de se délivrer. Mais c'est lorsque le sperme se coagule en se desséchant que se joue la tragédie. Mille petits grumeaux sont autant de pièges mortels. Voici quatre animalcules dont les appendices se sont agglutinés. Ils font des efforts désespérés pous s'affranchir : «On les voyoit, note Spallanzani, tantôt monter, tantôt descendre ; ils se tordoient à droite et à gauche, mais ils restèrent immobiles ". Un à un, ils réussissent à se libérer, mais pour se jeter contre d'autres grumeaux où les attend l'inéluctable [30]. Dans un autre ouvrage, Spallanzani s'emploie d'abord à réfuter Buffon en montrant que le comportement des animalcules obéit à des réflexes animaux. Il donne alors un tableau pittoresque de leurs mœurs : «On les voit, écrit-il, s'élancer en tous sens ; décrire dans l'eau, tantôt une ligne droite, tantôt une ligne oblique ; quelquefois se mouvoir circulairement et se jeter tous avec une égale activité sur les petits morceaux de matière qu'ils rencontrent dans leur route... Si quelques-uns se mettent à la poursuite de leurs camarades, bientôt ils les atteignent par une marche vive, s'arrêtent, tournent autour d'eux, et savent saisir une ouverture pour pénétrer dans le tissu de leur peau ». Certains « avoient le singulier instict de se réunir et de se rassembler vers une matière séminale dispersée ça et là dans plusieurs endroits de l'infusion, de manière qu'ils tournoient tout autour, et y sautilloint comme dans un endroit où il se plaisoient beaucoup, sans paroître se soucier de se transporter ailleurs. J'ai vû très sensiblement que nos petits animaux n'alloient dans cet endroit que pour y prendre quelques sucs nourriciers... $\gg[29]$.

Le point fort des recherches de Spallanzani repose toutefois sur la réfutation de l'expérience de Needham qui fier de ses idées et de ses pseudo-découvertes, se hâta de les communiquer à Buffon et plus tard à Spallanzani. Buffon accepta aussitôt les théories de 
Needham et les partagea sans les contrôler expérimentalement et en se contentant de soutenir que la cause productrice des êtres nés spontanément dans les infusions étaient les "particules organiques "; C'est ainsi que les idées de Needham et de Buffon se confondent et deviennent, comme l'écrira Spallanzani en 1765 " le système de la génération spontanée de Messieurs Needham et Buffon ». Spallanzani, en scientifique moderne, soutient d'abord, et pour la première fois semble-t-il, qu'on ne peut tirer aucune conclusion définitive d'une seule expérience : «Une ou deux observations, écrit-il, ne doivent pas suffire pour mériter les suffrages des philosophes éclairés ; on ne les obtient, ces suffrages, que par une chaîne de faits évidents, conformes entr'eux, et suivis avec l'attention la plus marquée ». Claude Bernard et Pasteur ne diront rien d'autre [9]. Voilà tout ce que fit Spallanzani pour démolir le « système de la génération spontanée de Needham et Buffon ", et son œuvre est allé bien au-delà du but qu'il se proposait, elle devint le point de départ de la biologie moderne [22].

\section{REFERENCES}

1. ANDROUTSOS G. : John Hunter (1728-1793) : fondateur de la chirurgie scientifique et précurseur de l'urologie. Progrès en Urologie, 1998, 8 : 1087-1096.

2. ARISTOTE. : De la génération des animaux. Traduction de P. Louis, Paris, Les Belles Lettres, 1961 : 23-30.

3. BARIETY M., COURY CH.: Histoire de la Médecine. Paris, Arthème Fayard, 1963 : 518-520.

4. BONNET CH. : Euvres d'histoire naturelle et de philosophie. 17 vol. Considérations sur les corps organisés (t.5), Neuchâtel, Fauche, 1774 : 242.

5. BUFFON G.L. : Histoire générale et particulière. 44 vol. Paris, Ménard et Desenne, 1749-1825, t.5 : 18.

6. CLIFFORD D. : Antony van Leeuzenhoek and his little animals. London, Staples Press Limited, 1932 : 13-16.

7. CRÉHANGE P.A. : Les livres anciens de médecine et de pharmacie. Paris, Les éditions de l'amateur, 1984 $: 108$.

8. CUVIER G. : Recherches sur les ossements fossiles des quadrupèdes. 4 vol. Paris, Déterville, 1812, t.2 : 232.

9. DARMON P. : L'homme et les microbes. Paris, Arthème Fayard, 1999 : 66-78.

10. DE GRAAF REINIER, : On the human reproductive organs. Journal of Reproduction and Fertility, suppl.,17,1972.

11. FRYDMAN R. : Dieu, la médecine et l'embryon, Paris, Odile Jacob, 1999 : 24-28.

12. GONZALÈS J. : Histoire naturelle et artificielle de la procréation. Paris, Bordas, 1996 : 221-225.

13. ARTSOECKER N. : Essay de Dioptrique. Paris, BNP, $1694: 28-34$

14. HARVEY W. : De Generatione animalium. London, Royal College of Physicians, 1651 : 42-47.

15. JACQUARD A.L. : La légende de la vie. Paris, Flammarion, 1992 : 105-112.

16. KERCKRING T. : Spicilegium anatomicum. Amsterdam, Andrea Frisi, 1670 : 27-30.

17. KÜSS R., GREGOIR W. : Histoire illustrée de l'urologie. Paris, Roger Dacosta, 1988 : 489-490.

18. MARTIN B. : Researches in Embryology. London, $1839: 35-37$.

19. MAYR E.R. : Histoire de la biologie, diversité, évolution et héredité. Paris, Arthème Fayard, 1989 : 611 614.

20. NETTER A., ROZENBAUM H. : Histoire illustrée de la contraception. Paris, Roger Dacosta, 1985 : 105.

21. PARÉ A. : Des monstres et prodiges. Genève, Droz, $1971: 32-34$.

22. PENSO G. : La conquête du monde invisible. Parasites et microbes à travers les siècles. Paris, Roger Dacosta, 1981 : 242-247.

23. PRÉVOST ET DUMAS. : Troisième mémoire sur la génération. Paris, $1824: 42$.

24. RÉAUMUR A. : Mémoires pour servir à l'Histoire des incectes. Paris, 1737, III : 37.

25. REDI F. : Esperienze intorno alla generazione degl' insetti. Florence, $1668:$ 19-23.

26. ROSTAND J. : Sciences et génération. Paris, Fasquelle, $1940: 19-20$.

27. ROSTAND J. : Les origines de la biologie expérimentale et l'abbé Spallanzani. Paris, Fasquelle, 1951 : 28-30.

28. ROSTAND J. : Des grenouilles et des hommes. Allocution présidentielle prononcée devant la Société zoologique de France, Juin 1963.

29. SPALLANZANI L. : Nouvelles recherches sur les découvertes microscopiques et la génération des corps organisés avec notes de $M$. de Needham, Londres et Paris, s.d., 2 t. en 1 vol., Paris, 1760 : 4445.

30. SPALLANZANI L. : Observations d'expériences faites sur les animalcules des infusoires, Paris, Gauthier-Villars, coll. "Les maîtres de la pensée ", 2 vol. 
31. SPEERT H. : Histoire illustrée de la Gynécologie et de l' Obstétrique. Paris, Roger Dacosta, 1976 : 123.

32. SWAMMERDAM J. : Miraculum naturae, sive uteri muliebris fabrica. Lugdundi Batavorum, T. Haak and S. Luchtmans, $1729: 123-129$.

33. TÉTRY A. : Jean Rostand : Un homme du futur". Lyon, La Manufacture, 1988 : 121.

34. VALLISNERI A.: Considerazioni, ed Esperienze intorno alla Generazione de' Vermi Ordinari del Corpo umano. Padova, Manfré Giovanni, 1710 : 1618.

35. VON HALLER A.: Operum anatomia argumenti minorum. Lausannae, Francisci, Grasset, $1768:$ 4551.

36. WOLFF F.-K. : Theoria generationis. Thèse, Halle, 1759 .

\author{
ABSTRACT \\ Lazzaro Spallanzani (1729-1799) and \\ the first helped procreations \\ G. ANDROUTSOS, ST. GEROULANOS
}

In this article we approach briefly the main theories concerning the physiology of reproduction (ovism, spermism, preformation, epigenesis, spontaneous generation, pangenesis) through the ages. In addition, we present the first experimentations of artificial procreation, emphasizing on the role of Spallanzani in this matter.

Key words : Spallanzani, reproduction, assisted procreation, artificial insemination 